\title{
Quantitative microscopy and systems biology: seeing the whole picture
}

\author{
Peter J. Verveer · Philippe I. H. Bastiaens
}

Accepted: 17 September 2008 / Published online: 1 October 2008

(C) The Author(s) 2008. This article is published with open access at Springerlink.com

\begin{abstract}
Understanding cellular function requires studying the spatially resolved dynamics of protein networks. From the isolated proteins we can only learn about their individual properties, but by investigating their behavior in their natural environment, the cell, we obtain information about the overall response properties of the network module in which they operate. Fluorescence microscopy methods provide currently the only tools to study the dynamics of molecular processes in living cells with high temporal and spatial resolution. Combined with computational approaches they allow us to obtain insights in the reactiondiffusion processes that determine biological function on the scale of cells.
\end{abstract}

Keywords Fluorescence microscopy - Systems biology . Biochemical networks

\section{Introduction}

Large-scale studies such as the various genomics and proteomics projects have yielded a wealth of data. Thanks to these efforts we have identified most of the basic building blocks of life. Yet, with such a reductionist approach we are not able to fully predict how organisms develop and function. For this, we need to understand how the basic components of a cell or an organism interact and how functionality

\footnotetext{
P. J. Verveer · P. I. H. Bastiaens ( $\square)$

Department of Systemic Cell Biology,

Max Planck Institute of Molecular Physiology,

Otto-Hahn-Strasse 11, 44227 Dortmund, Germany

e-mail: philippe.bastiaens@mpi-dortmund.mpg.de

P. J. Verveer

e-mail: verveer@mpi-dortmund.mpg.de
}

emerges from the systems build from these components (Ideker 2004; Ideker et al. 2001; Kitano 2002a, b). Although the roots of systems biology go back to the first half of the 20th century (Weiner 1948), we have recently seen a surge of activity in this field, fueled by the wealth of molecular data that became available. At the same time, rapid development in the computational sciences have made it possible to simulate the complex spatial/temporal dynamics of large multi-component molecular systems. Not surprisingly, much work has focused on experimental data obtained by such techniques as DNA microarrays, gel electrophoresis, and mass spectrometry that were so successful in acquiring the data for large-scale genomic and proteomic projects. However, most of these technologies acquire data from a homogenized population of cells and do not offer spatial and temporal resolution at the scales at which these biological systems operate. Biological objects are spatially highly organized, and to fully investigate the spatial and dynamic behavior of such systems, they must be studied in single living cells or in living organisms. For this reason we turn to another field where remarkable progress has been made in recent years: biological imaging.

Imaging in biology goes back to the 17 th century when Antonie van Leeuwenhoek first observed single-celled organisms that are invisible to the naked eye. Since then, optical microscopy has been a mainstay of biology, mainly as a qualitative tool. In the last decades of the 20th century a flurry of activity in the field occurred, and critical technical advances, such as digital imaging, fluorescent proteins and confocal microscopy opened new possibilities for quantitative imaging. This made it possible to observe the spatial and temporal distributions of proteins inside living cells and organisms. More recently, important biochemical and biophysical parameters such as protein diffusion and intra-cellular molecular reactions have become observable 
in living cells via fluorescence microscopy. Thus, in addition to the typical phenotypic microscopy studies of biological systems that have been a mainstay of biology for centuries, functional studies have become possible, for instance to directly image biochemical reactions inside living cells (Wouters et al. 2001). The fluorescence microscopy methods that are now available are a perfect match for systems biology since they make it possible to study the spatial and dynamic properties of living systems with minimal perturbations. Therefore, it is not surprising that they are making their way into this field (Megason and Fraser 2007).

Below we shortly review systems biology methods and fluorescence microscopy separately and continue to describe how they can be integrated into a single workflow that is more than the sum of two individual approaches. As a case study, we describe how enzyme activity can be imaged quantitatively in living cells and how this was used in combination with mathematical modeling to reveal spatial regulation of growth factor signaling. As a second example, we discuss an outstanding question in cell biology: how is signaling mediated via protein nanoclusters in the plasma membrane? We will argue that a combination of computational biology methods with novel fluorescence imaging methods can bridge the scale between nanoscopic processes and cellular response.

\section{Systems biology}

Biological systems operate on various levels ranging from sub-cellular gene and protein networks to the collective behavior of cells within an organism. Generally they consist of complex networks of components that are tuned by evolution to perform their tasks in a robust manner. Biological functionality on the scale of cells does not arise from individual gene or gene products but from their complex interactions. Therefore, whereas the study of individual components such as genes and proteins is a crucial step, their collective behavior must be studied to understand cell functions. Here it is important to consider two important aspects of any biological system: the scale at which it operates, and its dynamic behavior. Principally, biological systems are multi-scale, and the different networks that can be defined interact with each other. For instance, genetic networks control protein production, thereby largely defining the signal transduction networks that operate in living organisms. These protein-based signaling networks ultimately feed back into the operation of genetic networks. Nevertheless, we will often consider networks as independent systems, for instance, many systems biology studies focus independently on gene regulatory networks or on signal transduction networks. This is often justified because the time-scales at which these networks operate can be very different. This points us to the second important property of biological systems, which is their dynamic behavior. Many of the functions that arise from these complex networks can only be understood when their dynamic properties are studied. That is, only by studying the dynamic response of the network to perturbations or stimuli its fundamental properties can be investigated. Only then we can start to understand complex biological processes at a cellular scale, such as chemotaxis, differentiation, cell division, or the development of organisms. Although the temporal properties of biological networks are often emphasized in systems biology, we would like to stress here that many biological phenomena show spatial features that are crucial to the function of the biological networks. An example is that of gradients of signaling activities that organize signaling around specific organelles, providing cues for such processes as cell division and cell motility (Bastiaens et al. 2006; Kholodenko 2006).

A system-level understanding of biological phenomena is not possible by intuitive exploration of experimental data, due to the inherent complexity of these systems. For this reason, computational methods have become one of the main tools of systems biology to analyze experimental data and test hypotheses (Kahlem and Birney 2006; Kitano 2002a). By building accurate mathematical models of the complex networks of interest, their behavior can be investigated and predicted. It should however be stressed that systems biology remains a data-driven field, with the goal to understand complex systems based on experimental data. Thus, in addition to mathematical modeling, it is important to design proper experiments to acquire suitable quantitative data, and to develop adequate data-mining methods. The experimental data can then be used to validate the mathematical models and vice-versa the mathematical model can be used to design further experiments. Thus it is critical to the systems biology approach that a tight iterative feedback loop is created between experimental and computational systems biology.

Experimental systems biology has often focused on large data sets, for instance of gene expression profiles generated using microarray chips, or of protein-protein interaction networks determined by two-hybrid technologies. Such data then form the basis for computational systems biology techniques that are used to mine these data and to finally build mathematical models that can be used to understand biological systems. For instance, such an approach can be used to uncover transcription regulatory networks in mammalian cells (Tan et al. 2008). An important aspect of experimental systems biology is the use of different experimental data sources that can be integrated by the methods of computational systems biology. For instance, Ishii et al. used system wide data on transcriptional, protein and metabolite levels 
to study the central carbon metabolism of Escherichia coli (Ishii et al. 2007). Such data sets provide the starting point for computational approaches that can be used to understand fundamental physiological processes. A second aspect is that experimental procedures ideally should provide spatially- and time-resolved dynamic data, needed to understand the dynamics of the systems with high spatial resolution within cells or organisms. This poses a technical challenge for many of the experimental methods that are currently employed, which are often limited to measuring a few low-resolution time-points after a perturbation or stimulation without any intra-cellular spatial resolution.

The computational side of systems biology has seen accelerated development in recent years. It comprises of several facets that are of equal importance. Firstly, it should be recognized that the acquisition of quantitative data generally requires sophisticated data analysis methods to extract quantitative parameters from raw experimental data. Secondly, especially in the case of large data sets, data mining methods become essential to reduce and extract important features as inputs for models. Thirdly, accurate models must be build that can be used to understand and predict the behavior of the biological system. These three aspects have existed long before in independent fields (for instance in physics, bioinformatics, and mathematical biology) but in systems biology where experimental data forms the basis of mathematical models of whole systems, it is especially important that they are well integrated (Kahlem and Birney 2006).

For both experimental and computational approaches in systems biology there is a wealth of methods available, drawing from various fields such as chemistry, physics, mathematics and engineering, and we would like to refer the reader to the literature for further details. We will now concentrate on the use of fluorescence microscopy methods in experimental systems biology and how data acquired by these methods can be used in computational systems biology.

\section{Advanced fluorescence microscopy}

Optical microscopy has long been an important tool in the biological sciences. Its role was reduced in the last century with the advent of molecular biology techniques, which focused much of the efforts in the biological fields away from intact organisms and cells towards molecular components and processes that were not accessible with the microscopy techniques of the time. Nevertheless, the 20th century was also an exciting time for microscopy, on the one hand with the development of entirely new technologies such as electron microscopy and scanning probe microscopy, and on the other hand by the rapid developments in optical microscopy. Here we will focus on fluores- cence microscopy, which due to its unique ability to specifically visualize molecular components in living cells has become prevalent in the biological sciences. In fluorescence microscopy, the molecule of interest is labeled with a chromophore that emits light when excited by light of the appropriate wavelength. This makes it possible to specifically visualize the localization of labeled molecules, in contrast to traditional optical microscopy where individual molecular components cannot be distinguished. Thus, the use of a specific label is the key advantage of fluorescence microscopy in biology. Initially fluorescence microscopy techniques relied on direct labeling with organic fluorophores that targeted particular components or organelles, or by using labeled antibodies to target specific molecular species. In most cases, these approaches could not be applied in living cells or organisms, and they do not allow the visualization of dynamic processes. The discovery and cloning of green fluorescent proteins (GFP, Chalfie et al. 1994; Tsien 1998) proved therefore to be a dramatic changing point for the application of optical microscopy in the biological sciences. Fluorescent proteins can be fused by molecular biology techniques to a protein of interest and the resulting construct can be expressed by various techniques in living cells or even whole animals. In many cases, the normal functions of the protein of interest are not disturbed by the fluorescent tag, and its localization and dynamic behavior can be followed in living cells or organisms on physiologically relevant time-scales. Recent years have brought new labeling technology and probes, such as quantum dots and in vivo chemical labeling techniques (for instance Flash/Rehash and related approaches, Marks and Nolan 2006).

The development of these specific labeling techniques made fluorescence microscopy a powerful tool for biology and drove the development of new microscopy methods, which increased its usefulness even further. Two landmark developments deserve particular attention as they marked the onset of quantitative fluorescence microscopy in modern biology: digital imaging allowed unprecedented processing and quantification possibilities and the introduction of confocal microscopy made quantitative high-resolution three-dimensional imaging of samples a reality. The use of sensitive cameras and other electronic detectors made it possible to record low-intensity signals with high sensitivity and store these as quantitative digital data. This made it possible to apply computational post-processing steps to improve and quantify the experimental data. The second landmark was the development of confocal microscopy that allowed acquisition of sharp in-focus images that are not contaminated by out-of-focus blur, which distorts the image and complicates quantitative analysis (Pawley 2006). Whereas conventional fluorescence microscopy remains an important tool, the development of confocal microscopy 
marked a trend toward instrumentation capable of quantitative measurements of physiological parameters in living cells and organisms. These technical developments continued throughout the last decade of the 20th century and are still continuing to these days. New tools such as two-photon microscopy (Helmchen and Denk 2005), which allows efficient three-dimensional imaging in whole organisms and total internal reflection microscopy (TIRF, Axelrod et al. 1983; Toomre and Manstein 2001), which allows specific observation of plasma membrane-resident proteins, have joined the confocal microscope. Currently the field is being invigorated with the development of various exciting technologies that allows fluorescence microscopy to approach molecular resolution (Betzig et al. 2006; Hell 2007; Rust et al. 2006). Meanwhile, also molecular processes in cells within intact organisms have become accessible with the development of two-photon microscopy and alternative approaches such as single plane illumination microscopy (SPIM, Huisken et al. 2004).

Many of these new developments focused on increasing the quality and resolution of fluorescence microscopy, which is of importance for phenotypic investigation of cellular objects. However, alternative fluorescence spectroscopy and microscopy techniques have also seen rapid development, and have made it possible to directly investigate biochemical processes inside cells. These developments often proceeded by incorporating modalities from the field of fluorescence spectroscopy into the microscope making them available to the biologist for use in living cells and organisms. Various forms of fluorescence resonance energy transfer (FRET, Wouters et al. 2001) microscopy have allowed the direct and quantitative detection of molecular interactions inside living cells. Protein diffusion can be observed by techniques such as fluorescence recovery after photobleaching (FRAP, Koppel et al. 1976; Sprague and McNally 2005), single fluorophore tracking (SFT, Peters 2007), or fluorescence correlation spectroscopy (FCS, Bacia et al. 2006; Haustein and Schwille 2007; Magde et al. 1972). Various techniques, employing GFP-based reporters, allow the detection of physiological parameters such as enzyme activities, $\mathrm{Ca}^{2+}$ concentrations or $\mathrm{pH}$ (Miyawaki 2003). With these developments it has become possible to observe not only the localization of cellular components, but also their physiological state, and their interactions at a molecular level.

These developments in fluorescence microscopy have led to a toolbox of techniques for localizing physiological parameters and reactions states that are now slowly becoming standard in the biological sciences. Table 1 gives a list of the most relevant developments in optical fluorescence microscopy together with their main advantages. These fluorescence microscopy techniques offer several key advantages that cannot be realized with the more traditional tools from biochemistry and molecular biology. First of all, measurements can now be carried out with high temporal and spatial resolution in intact living cells or organisms. Moreover, measurements are done on single cells, rather than on populations, making it possible to study stochastic effects, for instance noise in gene expression (McAdams and Arkin 1999; Raser and O'Shea 2005). Even in a clonal population of cells, noise in expression levels can lead to very different network behavior, for instance in the case of bistable networks that depend critically on the relative concentrations of components (Reynolds et al. 2003; Tyson et al. 2003). Armed with these tools, the biologist can now observe the dynamic distribution of molecular components and their interactions in the physiological context of living cells.

\section{Fluorescence microscopy and systems biology}

From our discussion above it is clear that fluorescence microscopy is a versatile tool for the specific observation and quantification of molecular components and reactions within single living cells and organisms. These are exactly the requirements for the acquisition of appropriate data for the analysis of the spatial and dynamic properties of biological networks. It is therefore not surprising that highthroughput fluorescence microscopy methods have been developed and employed for drug discovery and profiling (Mitchison 2005; Perlman et al. 2004), and for studying cellular processes (Neumann et al. 2006; Pelkmans et al. 2005; Pepperkok and Ellenberg 2006). The advantages are obvious (Megason and Fraser 2007), and there is no doubt that these approaches will become more and more available as routine tools for large-scale system biology projects. So far, the high-throughput microscopy methods that have been reported in the literature are in essence conventional imaging methods that rely on the observation of cellular phenotypes in response to RNA interference or chemical perturbations. To study the structure of the regulatory and signal transduction networks that govern function in intact living cells, the advanced fluorescence imaging methods mentioned above need to be translated to a high-throughput setting. This will make it possible to elucidate the logical topology (network structure) of large biochemical networks by imaging their response to chemical or genetic perturbations (Kholodenko et al. 2002; Santos et al. 2007).

In vitro studies of a single network component can only reveal individual molecular properties. In the physiologically relevant context of the cell, a network determines the behavior of each of its components. Therefore we learn about the properties of the whole network by observing the (reaction) dynamics of a single component by fluorescence microscopy. A good example of this is given by the switch- 


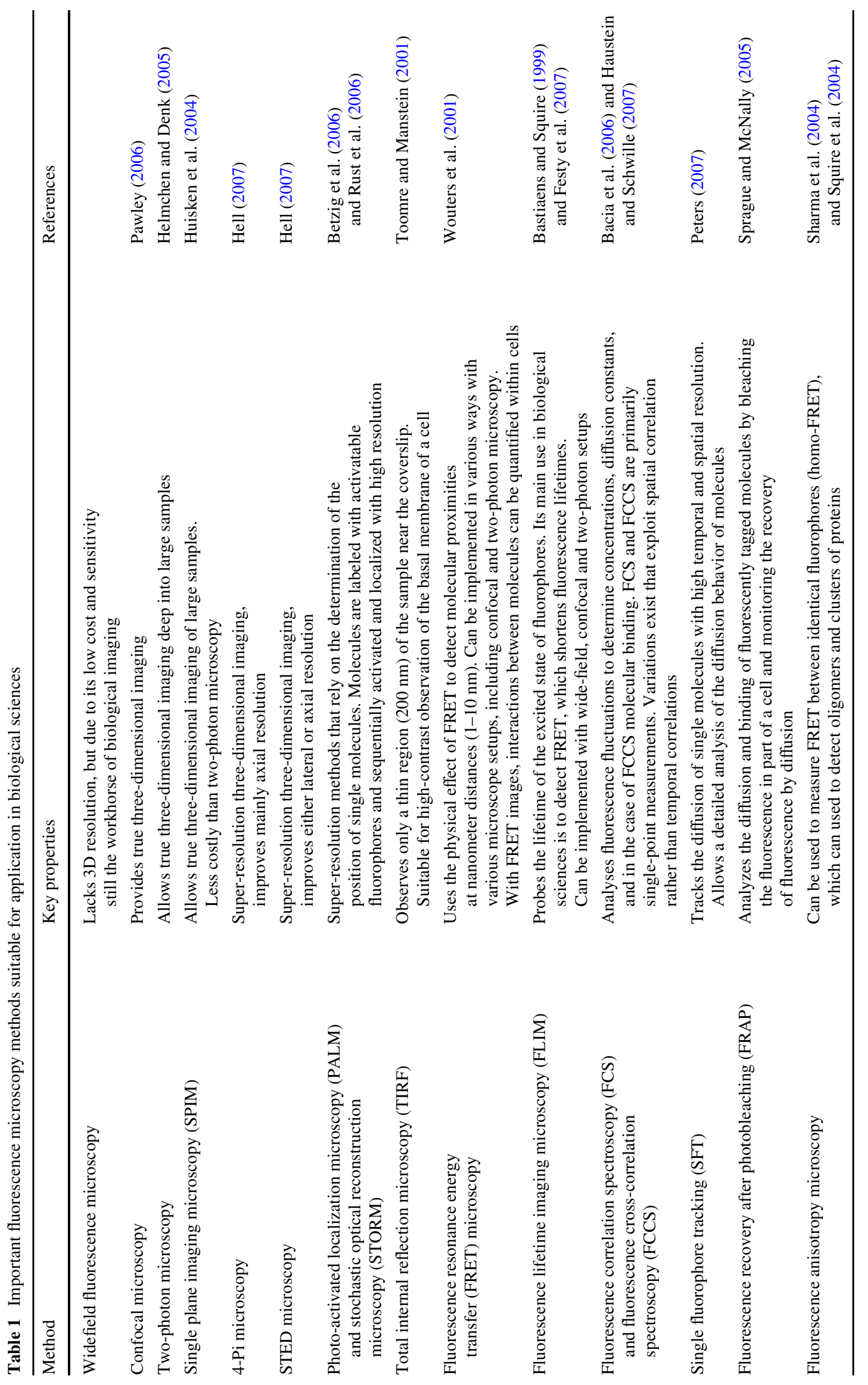


like response to growth-factor stimulation, which was studied extensively using FRET microscopy to quantitatively image the phosphorylation state of the epidermal growth factor receptor (EGFR). Initially it was found that EGRF activity could rapidly spread after focal stimulation in the absence of soluble ligand (Verveer et al. 2000b). Further studies combined with mathematical analysis revealed that a dynamic balance between receptor tyrosine kinase and phosphatase activity defines a bistable system that can be triggered into an active state even in the absence of ligand (Reynolds et al. 2003; Tischer and Bastiaens 2003). This early work is a good example of a study where quantitative fluorescence microscopy methods were used to study a spatial effect in single intact cells, finally revealing the dynamic properties of the underlying biochemical network. Such a network could not have been studied with conventional biochemical methods since variations in expression level could easily mask the switch-like behavior observed in single cells.

Fluorescence microscopy methods are able to provide a wealth of data on collective behavior of molecules in the physiological context of the living cell or organism. However, integrating such data to understand the dynamic behavior of molecular networks is non-trivial. In many cases, the experimental data still require a great deal of analysis and interpretation. In most cases no single fluorescence imaging method can provide sufficient information to understand the system, since currently no method can measure all needed parameters, such as interactions and diffusion, simultaneously at the appropriate spatial and temporal resolutions. The various types of fluorescence microscopy have however their individual strengths and can provide different pieces of the puzzle separately. The methods of computational systems biology provide the ideal tools to aggregate these data into an accurate and quantitative model. To illustrate this we describe in the next section two examples of problems where more traditional approaches have failed to provide a clear picture.

\section{Case studies}

The spatial regulation of growth factor signaling

An example of the use of a systems biology approach to uncover a spatially regulated signaling mechanism is given by the work of Yudushkin et al. (2007) on the phosphotyrosine phosphatase 1B (PTP1B). This enzyme resides on the surface of the endoplasmic reticulum (ER) (Frangioni et al. 1992), and terminates, among others, the signaling of the epidermal growth factor receptor (EGFR) along the surface of the ER (Haj et al. 2002). An important question is how the balance of kinase and phosphatase activities is spatially organized in the cell to regulate growth factor signaling. To tackle this question, the authors developed a new imaging approach to spatially resolve enzyme-substrate (ES) reactions and thereby monitor enzyme activity in living cells (Fig. 1a). By tagging PTP1B with GFP, and loading a chromophore-labeled synthetic phosphotyrosine-containing peptide as its substrate into cells, they could demonstrate a stable gradient of the ES complex of PTP1B and the substrate peptide (Fig. 1b) by quantitatively imaging fluorescence resonance imaging transfer (FRET) by fluorescence lifetime imaging microscopy (FLIM) (Verveer et al. 2000a, b). After uncaging a synthetic substrate with ultraviolet light, the gradient of ES complex established itself too slowly to be consistent with the high catalytic activity of PTP1B as measured in vitro. Furthermore, the existence of the stable ES-gradient was shown to be maintained by a dynamic phosphorylation/dephosphorylation cycle, where the synthetic peptide would not only be dephosphorylated by PTP1B, but also be (re)phosphorylated by EGFR.

In a kinase/phosphatase system, where the two activities are spatially separated, gradients can indeed form based on simple reaction-diffusion principles (Brown and Kholodenko 1999). In this case, the plasma membranebound EGFR and the ER-bound PTP1B are expected to generate a steady-state gradient of phosphorylated substrate, which will be reflected in a gradient of the ES complex. The authors explored this by formulating a reaction-diffusion model of the kinase-phosphatase reaction cycle. Indeed, they found that a gradient of phosphorylated substrate and of ES complex could be established, as long as the specific activity of PTP1B in vivo was orders of magnitude lower than in vitro.

However, upon further analysis, this simple model showed a high sensitivity to the relative levels of phosphatase and kinase (Fig. 1c), and thus lacked robustness that is often observed in biological systems. Indeed, such sensitivity was inconsistent with the data: in cells the ES gradient was persistently observed, independent of observed variability of concentrations and other parameters, such as cell shape. Moreover, the gradient remained stable upon EGFR activation by EGF, which increases its kinase activity by up to a factor of five. The question was which mechanism could be responsible for this robustness. The observation that the catalytic activity of PTP1B in vivo was much lower than expected from in vitro data provided the answer to this question. By introducing a gradient of intrinsic PTP1B activity, where activity is much lower near the periphery of the cell than in the perinuclear region, a model was obtained that was robust to variations in the relative concentrations of kinase and phosphatase (Fig. 1d). Based on this theoretical analysis, the authors reasoned that their data could only be explained if the intrinsic catalytic activity of PTP1B is spatially regulated to be much lower at the cell 
A
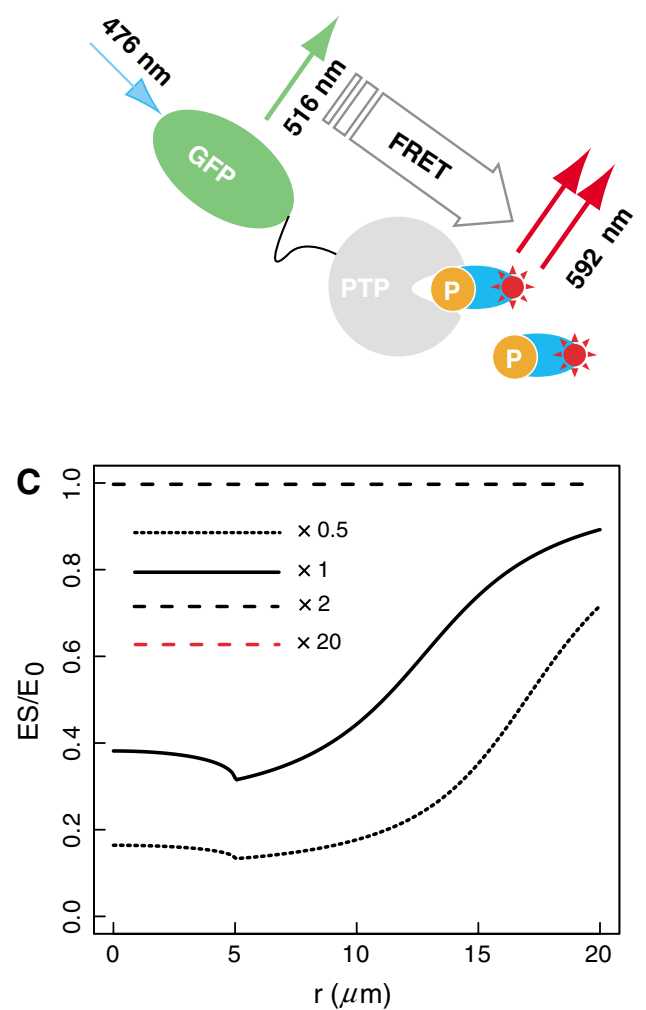

Fig. 1 Spatial regulation of growth factor signaling. a The interaction of a GFP-tagged enzyme PTP1B with a labeled, phosphorylated substrate can be measured by FRET. b Fraction of enzyme in complex with the substrate $\left(\mathrm{ES} / E_{0}\right)$ as measured by FLIM. $\mathrm{c} \mathrm{ES} / E_{0}$ as a function of the distance from the nucleus for several relative values of the catalytic activity of the kinase assuming that the catalytic activity of

periphery. This model makes biological sense if one considers the much higher catalytic rate of PTP1B as compared to EGFR: the membrane-proximal activity of PTP1B is maintained at a sufficiently low level to permit signal transmission in the cell via endocytosis of phosphorylated EGFR, but kept sufficiently high to suppress EGFR activity in the absence of growth factor stimuli. The growth factor signal is terminated by endocytic trafficking of phosphorylated EGFR to the high-activity pool of PTP1B in the perinuclear region.

This work is a clear example of the application of advanced fluorescence imaging and mathematical modeling techniques to study the spatial regulation of a signaling system inside living cells. Without mathematical modeling it would not have been possible to predict the properties of this system, notably the spatial regulation of PTP1B catalytic activity. This also demonstrates that the spatio-temporal properties of a regulatory network are observed via one of its crucial components within the cellular context. Although technically challenging, the ES imaging method is highly generic and therefore an excellent candidate to be
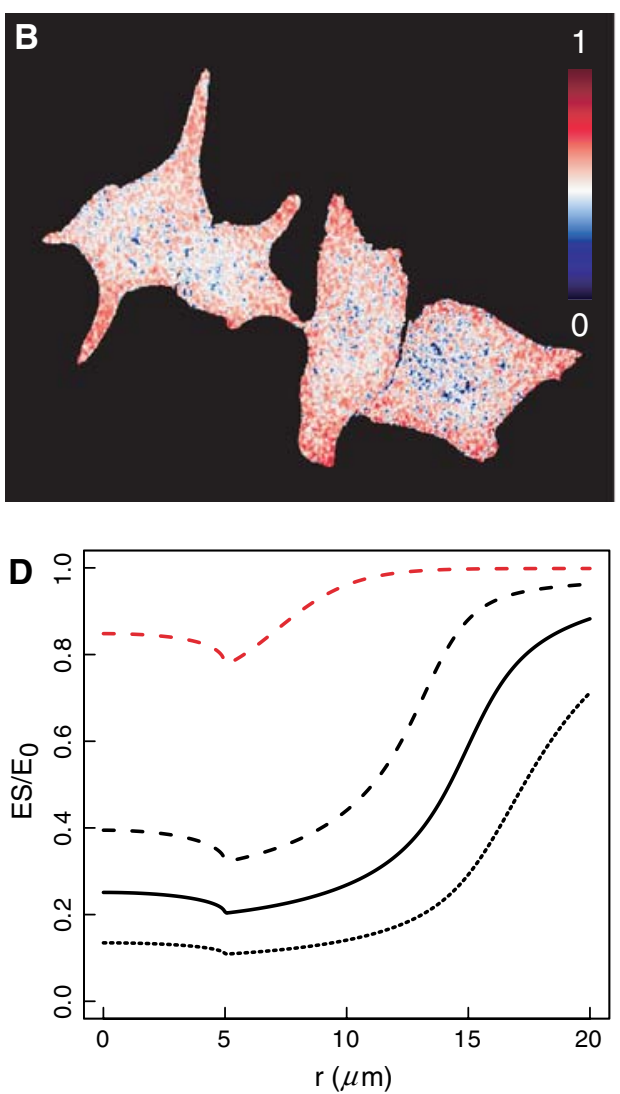

PTP1B is constant. d ES/ $E_{0}$ as a function of the distance from the nucleus for several relative values of the catalytic activity of the kinase assuming that the catalytic activity of PTP1B is spatially regulated, such that PTP1B activity is high near the nucleus and low near the periphery of the cell. The figure is adapted from (Yudushkin et al. 2007)

scaled up in a high-throughput microscope system to study the causality between enzymatic activities to reveal network structure in living cells.

Signaling via protein clusters in the plasma membrane

It is now widely accepted that biological membranes are highly heterogeneous environments where the translational diffusion of proteins is restricted by various mechanisms (Vereb et al. 2003). A good example is the clustering of molecules in membranes by mechanisms that may involve lipid-lipid, protein-protein and protein-lipid interactions. The best know examples of such a phenomenon are lipid rafts that are defined as small cholesterol-dependent domains in membranes in which certain proteins may aggregate (Lai 2003; Simons and Toomre 2000; Vereb et al. 2003). It has been suggested that such domains may play a crucial role in signal transduction by sorting the signaling molecules and modifying their output properties by increasing their local concentrations (Lai 2003; Simons and Toomre 2000; Vereb et al. 2003). Lipid rafts have been 
studied extensively, but a clear consensus on their nature and their role in cellular signaling has not been reached yet. This has been mainly due to a lack of proper experimental tools to study the formation and regulation of protein clusters at sub-micron spatial and millisecond temporal scales (Jacobson et al. 2007). Biochemical methods were initially used that provided limited information about the physical properties of lipid rafts, and only recently have novel fluorescence microscopy methods allowed the study of such small and fast phenomena in living cells. A prime example is the work of Sharma et al. who used fluorescence anisotropy imaging to show that GPI-anchored proteins form small cholesterol-dependent clusters (Sharma et al. 2004). Interestingly, their data indicate that these clusters are dynamically maintained in the cell in contrast to lipid domains that have been studied in reconstituted membrane systems. This work is an excellent example of the study of a phenomenon that can best be studied in living cells using functional microscopy methods. Indeed, we expect that microspectroscopic methods are the main tools that will be used to observe protein cluster formation in the plasma membrane and elucidate their function in signaling. This will require further development of the optical tools, but also will require the development of appropriate computational methods to interpret and integrate the experimental results into coherent models. A complex non-homogenous spatial organization of signaling molecules in the plasma membrane can be expected to lead to intricate signaling behavior: a typical case for a systems biology approach where various experimental methods must be combined with mathematical modeling.

The complexities of protein partitioning and its possible consequences are clearly demonstrated by the paradigm example of Ras, a small regulatory GTPase that has a pivotal role in many signaling pathways and in tumor development. The isoforms of Ras show a high degree of homology, but have distinct signaling outputs (Yan et al. 1998). These isoforms of Ras differ mainly in their C-terminal membrane anchors that determine cellular localization and micro-localization within membranes (Rocks et al. 2006). For instance, within the whole cell an acylation cycle dynamically partitions H-Ras between the plasma membrane and the Golgi (Rocks et al. 2005). On the other hand K-Ras is not subject to such an acylation cycle but its association with the plasma membrane is regulated via phosphorylation (Bivona et al. 2006). It is now well established that the compartmentalization of Ras in the cell leads to different signaling outputs and that individual pools of Ras, such as the Golgi-localized pool, form independent signaling entities (Mor and Philips 2006; Rocks et al. 2006). Clearly, the role of the various Ras isoforms in signaling networks is critically influenced by their localization at a cellular scale.
The isoforms of Ras are thought to also show differences in their localization at a nanometer scale in membranes. Experimental data suggest that the different Ras isoforms cluster in small dynamically maintained domains depending on various mechanisms (see Fig. 2). One line of evidence comes from data acquired by electron microscopy methods (Plowman et al. 2005; Prior et al. 2003). The dynamics of Ras in the plasma membrane have also been measured by single fluorophore tracking (SFT) (Lommerse et al. 2003, 2005; Murakoshi et al. 2004) and with fluorescence recovery after photobleaching (FRAP) (Niv et al. 1999, 2002). According to these data, H-Ras clusters into cholesterol dependent nanoscale domains in its inactive GDP-bound state. However, in the GTP-bound active state, $\mathrm{H}$-Ras clusters outside membrane rafts with the involvement of the $\beta$-galactoside-binding protein Galectin-1 (Hancock and Parton 2005). K-Ras on the other hand was shown to form cholesterol-independent clusters regardless of its activation state, with the involvement of another galectin, Galectin-3 (Hancock and Parton 2005). In addition, varying degrees of Ras compartmentalization within the plasma membrane by the actin cytoskeleton have been observed (Kusumi et al. 2005; Kusumi and Suzuki 2005). In spite of this broad variety of available data, a comprehensive model of dynamic Ras clustering and its regulation is not yet available. This is likely due to the lack of combined high temporal and spatial resolution in the experimental approaches that were employed. We expect that new imaging approaches, such as super-resolution methods (Betzig et al. 2006; Hell 2007; Rust et al. 2006) that combine these dimensions at appropriate scales, will complement existing

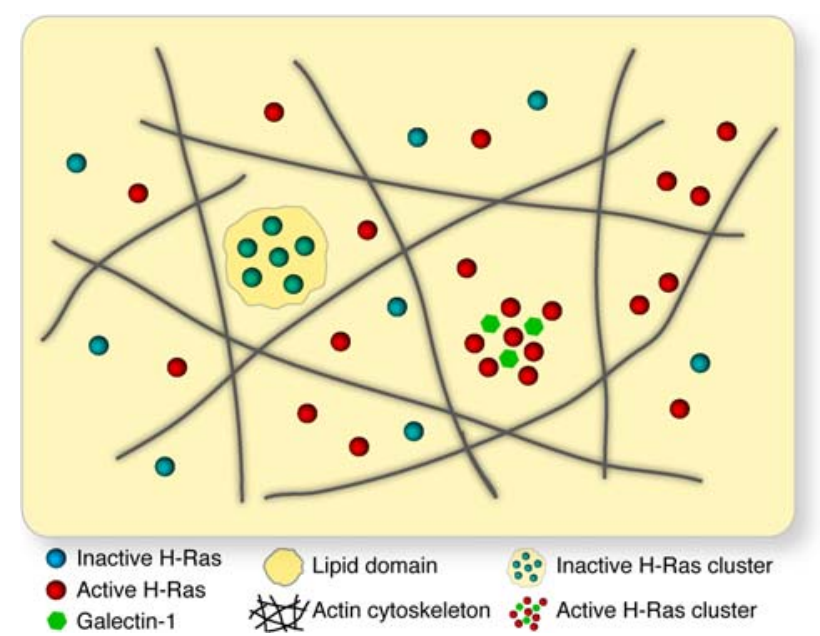

Fig. 2 H-Ras is distributed non-homogeneously in the plasma membrane. A significant fraction of the Ras molecules form small clusters, whereas the remainder is dispersed as monomers. Inactive H-Ras molecules are found in cholesterol-dependent domains. Active H-Ras forms clusters that depend on the Galectin- 1 scaffold. In addition, the diffusion of H-Ras is constrained by the actin cytoskeleton 
data to provide a more comprehensive picture of Ras partitioning.

In order to understand the role that Ras partitioning has in signaling, it will be necessary to observe interactions of the appropriate subset of Ras molecules with Ras effectors directly in living cells. Indeed differential activation of Ras between raft and non-raft domains has been observed by FRET microscopy (Fukano et al. 2007). SFT experiments have shown that Raf proteins bind to immobilized Ras-GTP molecules (Murakoshi et al. 2004) and EM analysis shows co-clustering of Raf and Ras-GTP (Plowman et al. 2005), suggesting that Raf binds to clustered active Ras. One can speculate that confinement and clustering of Ras and its effector molecules facilitates interactions with further components of a relevant signaling network. Mathematical modeling shows that this indeed could be the case (Nicolau et al. 2006), but this hypothesis awaits experimental confirmation. Alternatively, the clustering of Ras could confer beneficial response properties onto the signaling network. Tian et al. explored this possibility by using a mathematical model that coupled nanocluster properties with response properties of cytoplasmic signaling networks to show that the confinement of Raf molecules to active Ras clusters could lead to high-fidelity signal transduction (Tian et al. 2007). This work thereby represents the first step to bridge the gap between spatial temporal properties of Ras on nanoscales and the phenotypic response on a much larger cellular scale.

With the help of fluorescence microscopy methods that have sub-micrometer spatial resolution and sub-second temporal resolution, we are now beginning to understand the role of the three-dimensional cellular distribution of Ras in signal transduction. Likewise, investigating the significance of Ras nano-clustering on signal transduction can only be achieved by experimental methods that probe these systems on the nanometer spatial scale and the microsecond temporal scale. At this stage, reaction-diffusion models need to be developed that integrate the spatial and temporal information from these different scales to better understand signaling systems that contain Ras.

\section{Conclusion}

Understanding complex cellular systems requires understanding their temporal and spatial dynamics, not just their components. Molecules such as PTP1B and Ras are examples of crucial nodes in growth factor signaling networks. An important point that we wanted to stress here is that these molecules become reporters for the dynamic properties of the whole network when their activity is studied in the context of the cell. By isolating and studying them in vitro we can only learn about their individual molecular properties, but by investigating their behavior in their natural environment we obtain information about the collective behavior of signaling networks in which they operate.

Fluorescence microscopy methods provide the signal specificity to study the dynamics of molecular processes in living cells with high temporal and spatial resolution. Likewise, it can be expected that functional imaging will further develop to image molecular processes within cells of complete organisms (Weissleder and Ntziachristos 2003). Functional fluorescence microscopy is also suitable in a highthroughput setting and it can be expected that this will play a significant role in elucidating the causality in biochemical network structure in the near future by directly probing protein reaction states in living cells.

Acknowledgments We thank Ali Kinkhabwala for valuable comments on this review.

Open Access This article is distributed under the terms of the Creative Commons Attribution Noncommercial License which permits any noncommercial use, distribution, and reproduction in any medium, provided the original author(s) and source are credited.

\section{References}

Axelrod D, Thompson NL, Burghardt TP (1983) Total internal inflection fluorescent microscopy. J Microsc 129:19-28

Bacia K, Kim SA, Schwille P (2006) Fluorescence cross-correlation spectroscopy in living cells. Nat Methods 3:83-89

Bastiaens P, Caudron M, Niethammer P, Karsenti E (2006) Gradients in the self-organization of the mitotic spindle. Trends Cell Biol 16:125-134

Bastiaens PI, Squire A (1999) Fluorescence lifetime imaging microscopy: spatial resolution of biochemical processes in the cell. Trends Cell Biol 9:48-52

Betzig E, Patterson GH, Sougrat R, Lindwasser OW, Olenych S, Bonifacino JS, Davidson MW, Lippincott-Schwartz J, Hess HF (2006) Imaging intracellular fluorescent proteins at nanometer resolution. Science 313:1642-1645

Bivona TG, Quatela SE, Bodemann BO, Ahearn IM, Soskis MJ, Mor A, Miura J, Wiener HH, Wright L, Saba SG, Yim D, Fein A, Castro IPd, Li C, Thompson CB, Cox AD, Philips MR (2006) PKC regulates a farnesyl-electrostatic switch on K-Ras that promotes its association with Bcl-XL on mitochondria and induces apoptosis. Mol Cell 21:481-493

Brown GC, Kholodenko BN (1999) Spatial gradients of cellular phospho-proteins. FEBS Lett 457:452-454

Chalfie M, Tu Y, Euskirchen G, Ward WW, Prasher DC (1994) Green fluorescent protein as a marker for gene expression. Science 263:802-805

Festy F, Ameer-Beg SM, Ng T, Suhling K (2007) Imaging proteins in vivo using fluorescence lifetime microscopy. Mol Biosyst 3:381391

Frangioni JV, Beahm PH, Shifrin V, Jost CA, Neel BG (1992) The nontransmembrane tyrosine phosphatase PTP-1B localizes to the endoplasmic reticulum via its 35 amino acid $C$-terminal sequence. Cell 68:545-560

Fukano T, Sawano A, Ohba Y, Matsuda M, Miyawaki A (2007) Differential Ras activation between caveolae/raft and non-raft microdomains. Cell Struct Funct 32:9-15 
Haj FG, Verveer PJ, Squire A, Neel BG, Bastiaens PIH (2002) Imaging sites of receptor dephosphorylation by PTP1B on the surface of the endoplasmic reticulum. Science 295:1708-1711

Hancock JF, Parton RG (2005) Ras plasma membrane signalling platforms. Biochem J 389:1-11

Haustein E, Schwille P (2007) Fluorescence correlation spectroscopy: novel variations of an established technique. Annu Rev Biophys Biomol Struct 36:151-169

Hell SW (2007) Far-field optical nanoscopy. Science 316:1153-1158

Helmchen F, Denk W (2005) Deep tissue two-photon microscopy. Nat Methods 2:932-940

Huisken J, Swoger J, Bene FD, Wittbrodt J, Stelzer EHK (2004) Optical sectioning deep inside live embryos by selective plane illumination microscopy. Science 305:1007-1009

Ideker T (2004) Systems biology 101-what you need to know. Nat Biotechnol 22:473-475

Ideker T, Galitski T, Hood L (2001) A new approach to decoding life: systems biology. Annu Rev Genomics Hum Genet 2:343-372

Ishii N, Nakahigashi K, Baba T, Robert M, Soga T, Kanai A, Hirasawa T, Naba M, Hirai K, Hoque A, Ho PY, Kakazu Y, Sugawara K, Igarashi S, Harada S, Masuda T, Sugiyama N, Togashi T, Hasegawa M, Takai Y, Yugi K, Arakawa K, Iwata N, Toya Y, Nakayama Y, Nishioka T, Shimizu K, Mori H, Tomita M (2007) Multiple high-throughput analyses monitor the response of E. coli to Perturbations. Science 316:593-597

Jacobson K, Mouritsen OG, Anderson RGW (2007) Lipid rafts: at a crossroad between cell biology and physics. Nat Cell Biol 9:7-14

Kahlem P, Birney E (2006) Dry work in a wet world: computation in systems biology. Mol Syst Biol 2:1-4

Kholodenko BN (2006) Cell-signalling dynamics in time and space. Nat Rev Mol Cell Biol 7:165-176

Kholodenko BN, Kiyatkin A, Bruggeman FJ, Sontag E, Westerhoff HV, Hoek JB (2002) Untangling the wires: a strategy to trace functional interactions in signaling and gene networks. Proc Natl Acad Sci USA 99:12841-12846

Kitano H (2002a) Computational systems biology. Nature 420:206210

Kitano H (2002b) Systems biology: a brief overview. Science 295:1662-1664

Koppel DE, Axelrod D, Schlessinger J, Elson EL, Webb WW (1976) Dynamics of fluorescence marker concentration as a probe of mobility. Biophys J 16:1315-1329

Kusumi A, Nakada C, Ritchie K, Murase K, Suzuki K, Murakoshi H, Kasai RS, Kondo J, Fujiwara T (2005) Paradigm shift of the plasma membrane concept from the two-dimensional continuum fluid to the partitioned fluid: high-speed single-molecule tracking of membrane molecules. Annu Rev Biophys Biomol Struct 34:351-378

Kusumi A, Suzuki K (2005) Toward understanding the dynamics of membrane-raft-based molecular interactions. Biochim Biophys Acta 1746:234-251

Lai EC (2003) Lipid rafts make for slippery platforms. J Cell Biol $162: 365-370$

Lommerse PHM, Blab GA, Cognet L, Harms GS, Snaar-Jagalska BE, Spaink HP, Schmidt T (2003) Single-molecule imaging of the Hras membrane-anchor reveals domains in the cytoplasmic leaflet of the cell membrane. Biophys J 86:609-616

Lommerse PHM, Snaar-Jagalska BE, Spaink HP, Schmidt T (2005) Single-molecule diffusion measurements of $\mathrm{H}$-Ras at the plasma membrane of live cells reveal microdomain localization upon activation. J Cell Sci 118:1799-1809

Magde D, Elson E, Webb WW (1972) Thermodynamic fluctuations in a reacting system: measurement by fluorescence correlation spectroscopy. Phys Rev Lett 29:705-708

Marks KM, Nolan GP (2006) Chemical labeling strategies for cell biology. Nat Methods 3:591-596
McAdams HH, Arkin A (1999) It's a noisy business! Genetic regulation at the nanomolar scale. Trends Genet 15:65-69

Megason SG, Fraser SE (2007) Imaging in systems biology. Cell 130:784-795

Mitchison TJ (2005) Small-molecule screening and profiling by using automated microscopy. Chembiochem 6:33-39

Miyawaki A (2003) Fluorescence imaging of physiological activity in complex systems using GFP-based probes. Curr Opin Neurobiol 13:591-596

Mor A, Philips MR (2006) Compartmentalized Ras/MAPK signaling. Annu Rev Immunol 24:771-800

Murakoshi H, Iino R, Kobayashi T, Fujiwara T, Ohshima C, Yoshimura A, Kusumi A (2004) Single-molecule imaging analysis of Ras activation in living cells. Proc Natl Acad Sci USA 101:73177322

Neumann B, Held M, Liebel U, Erfle H, Rogers P, Pepperkok R, Ellenberg J (2006) High-throughput RNAi screening by time-lapse imaging of live human cells. Nat Methods 3:385-390

Nicolau DV, Burrage K, Parton RG, Hancock JF (2006) Identifying optimal lipid raft characteristics required to promote nanoscale protein-protein interactions on the plasma membrane. Mol Cell Biol 26:313-323

Niv H, Gutman O, Henis YI, Kloog Y (1999) Membrane interactions of a constitutively active GFP-Ki-Ras 4B and their role in signaling. Evidence from lateral mobility studies. J Biol Chem 274:1606-1613

Niv H, Gutman O, Kloog Y, Henis YI (2002) Activated K-Ras and HRas display different interactions with saturable nonraft sites at the surface of live cells. J Cell Biol 157:865-872

Pawley JB (2006) Handbook of biological confocal microscopy, 3rd edn edn. Springer, New York

Pelkmans L, Fava E, Grabner H, Hannus M, Habermann B, Krausz E, Zerial M (2005) Genome-wide analysis of human kinases in clathrin- and caveolae/raft-mediated endocytosis. Nature 436:78 86

Pepperkok R, Ellenberg J (2006) High-throughput fluorescence microscopy for systems biology. Nat Rev Mol Cell Biol 7:690696

Perlman ZE, Slack MD, Feng Y, Mitchison TJ, Wu LF, Altschuler SJ (2004) Multidimensional drug profiling by automated microscopy. Science 306:1194-1198

Peters R (2007) Single-molecule fluorescence analysis of cellular nanomachinery components. Annu Rev Biophys Biomol Struct

Plowman SJ, Muncke C, Parton RG, Hancock JF (2005) H-ras, K-ras, and inner plasma membrane raft proteins operate in nanoclusters with differential dependence on the actin cytoskeleton. Proc Natl Acad Sci USA 102:15500-15505

Prior IA, Muncke C, Parton RG, Hancock JF (2003) Direct visualization of Ras proteins in spatially distinct cell surface microdomains. J Cell Biol 160:165-170

Raser JM, O'Shea EK (2005) Noise in gene expression: origins, consequences, and control. Science 309:2010-2013

Reynolds AR, Tischer C, Verveer PJ, Rocks O, Bastiaens PIH (2003) EGFR activation coupled to inhibition of tyrosine phosphatases causes lateral signal propagation. Nat Cell Biol 5:447-453

Rocks O, Peyker A, Bastiaens PIH (2006) Spatio-temporal segregation of Ras signals: one ship, three anchors, many harbors. Curr Opin Cell Biol 18:351-357

Rocks O, Peyker A, Kahms M, Verveer PJ, Koerner C, Lumbierres M, Kuhlmann J, Waldmann H, Wittinghofer A, Bastiaens PIH (2005) An acylation cycle regulates localization and activity of palmitoylated Ras isoforms. Science 307:1746-1752

Rust MJ, Bates M, Zhuang X (2006) Sub-diffraction-limit imaging by stochastic optical reconstruction microscopy (STORM). Nat Methods 3:793-795 
Santos SDM, Verveer PJ, Bastiaens PIH (2007) Growth factor-induced MAPK network topology shapes Erk response determining PC12 cell fate. Nat Cell Biol 9:324-330

Sharma P, Varma R, Sarasij RC, Ira, Gousset K, Krishnamoorthy G, Rao M, Mayor S (2004) Nanoscale organization of multiple GPIanchored proteins in living cell membranes. Cell 116:577-589

Simons K, Toomre D (2000) Lipid rafts and signal transduction. Nat Rev Mol Cell Biol 1:31-39

Sprague BL, McNally JG (2005) FRAP analysis of binding: proper and fitting. Trends Cell Biol 15:84-91

Squire A, Verveer PJ, Rocks O, Bastiaens PIH (2004) Red-edge anisotropy microscopy enables dynamic imaging of homo-FRET between green fluorescent proteins in cells. J Struct Biol 147:62-69

Tan K, Tegner J, Ravasi T (2008) Integrated approaches to uncovering transcription regulatory networks in mammalian cells. Genomics 91:219-231

Tian T, Harding A, Inder K, Plowman S, Parton R, Hancock J (2007) Plasma membrane nanoswitches generate high-fidelity Ras signal transduction. Nat Cell Biol 9:905-914

Tischer C, Bastiaens PIH (2003) Lateral phosphorylation propagation: an aspect of feedback signalling? Nat Rev Mol Cell Biol 4:971974

Toomre D, Manstein DJ (2001) Lighting up the cell surface with evanescent wave microscopy. Trends Cell Biol 11:298-303

Tsien RY (1998) The green fluorescent protein. Annu Rev Biochem 67:509-544
Tyson JJ, Chen KC, Novak B (2003) Sniffers, buzzers, toggles and blinkers: dynamics of regulatory and signaling pathways in the cell. Curr Opin Cell Biol 15:221-231

Vereb G, Szöllosi J, Matkó J, Nagy P, Farkas T, Vigh L, Mátyus L, Waldmann TA, Damjanovich S (2003) Dynamic, yet structured: the cell membrane three decades after the Singer-Nicolson model. Proc Natl Acad Sci USA 100:8053-8058

Verveer PJ, Squire A, Bastiaens PI (2000a) Global analysis of fluorescence lifetime imaging microscopy data. Biophys J 78:21272137

Verveer PJ, Wouters FS, Reynolds AR, Bastiaens PI (2000b) Quantitative imaging of lateral ErbB1 receptor signal propagation in the plasma membrane. Science 290:1567-1570

Weiner N (1948) Cybernetics or control and communication in the animal and the machine. MIT Press, Cambridge

Weissleder R, Ntziachristos V (2003) Shedding light onto live molecular targets. Nat Med 9:123-128

Wouters FS, Verveer PJ, Bastiaens PI (2001) Imaging biochemistry inside cells. Trends Cell Biol 11:203-211

Yan J, Roy S, Apolloni A, Lane A, Hancock JF (1998) Ras isoforms vary in their ability to activate Raf-1 and phosphoinositide 3-kinase. J Biol Chem 273:24052-24056

Yudushkin IA, Schleifenbaum A, Kinkhabwala A, Neel BG, Schultz C, Bastiaens PIH (2007) Live-cell imaging of enzyme-substrate interaction reveals spatial regulation of PTP1B. Science 315:115119 